\title{
Spatial Vagueness and Imprecision in Databases
}

\author{
Alejandro Pauly \& Markus Schneider \\ Computer and Information Sciences and Engineering Dept. \\ University of Florida \\ Gainesville, FL, USA \\ \{apauly, mschneid\}@cise.ufl.edu
}

\begin{abstract}
The impossibility of current spatial database systems and GIS to handle spatial vagueness and imprecision has been recognized as an important problem in the spatial database domain. For years, researchers have focused on identifying the appropriate concepts that are necessary to effectively and efficiently deal with the vagueness and imprecision that not seldomly appears, but is widespread amongst spatial objects (especially those that are naturally occurring such as forests, mountains, and rivers). In this paper, we analyze the most popular formalisms for dealing with spatial uncertainty in databases. The analysis in this paper is centered around the definition of our Vague Spatial Algebra (VASA) which exemplifies a complete approach to handle vagueness in spatial databases. We compare VASA with existing concepts based on rough set theory and fuzzy set theory.
\end{abstract}

\section{INTRODUCTION}

For all available spatial data models in GIS and spatial database systems, the illusory assumption is made that all spatial objects can be adequately represented as exclusively crisp and exactly determined entities. That is, they implicitly assume that the position of points, the location and routes of lines, and the extent and hence the boundary of regions are precisely determined and universally recognized. Examples are man-made spatial objects (e.g., monuments, highways, buildings) and immaterial spatial objects (e.g., countries, districts, land parcels with their political, administrative, and cadastral boundaries). We denote these entities as crisp or determinate spatial objects.

On the other hand, for many geographic applications this premise is unfounded and inappropriate since the feature of spatial vagueness or spatial indeterminacy is inherent to their data. Positions of points turn out to be not exactly known, the locations and routes of lines are unclear, and

\footnotetext{
*This work was partially supported by the National Science Foundation under grant number NSF-CAREER-IIS0347574 .
}

Permission to make digital or hard copies of all or part of this work for personal or classroom use is granted without fee provided that copies are not made or distributed for profit or commercial advantage and that copies bear this notice and the full citation on the first page. To copy otherwise, to republish, to post on servers or to redistribute to lists, requires prior specific permission and/or a fee.

SAC'08 March 16-20, 2008, Fortaleza, Ceará, Brazil

Copyright 2008 ACM 978-1-59593-753-7/08/0003 ...\$5.00. regions do not have sharp boundaries, or their boundaries cannot be sharply determined. Examples are social or natural phenomena like terrorists' refuges and escape routes, population density, unemployment rate, soil quality, vegetation, and oil fields. We denote these entities as vague or indeterminate spatial objects.

Many efforts have been under way in the past years to develop a comprehensive model for handling spatial vagueness. These efforts, roughly classified in four categories according to their mathematical foundations, have so far failed to provide the necessary concepts and, more importantly implementations for handling spatial uncertainty in databases. In this paper, we analyze these efforts and draw conclusions that lead us to develop our own data model for spatial vagueness. Our model, denoted as the Vague Spatial Algebra (VASA), leverages existing concepts for crisp spatial data to define vague spatial objects, their operations, and predicates.

Section 2 summarizes the most important features of existing approaches to handling spatial vagueness. We follow in Section 3 with a description of VASA. Section 4 analyzes the advantages and disadvantages of VASA against the other approaches previously introduced. Finally, in Section 5, we provide conclusions and mention future work.

\section{APPROACHES FOR HANDLING SPATIAL UNCERTAINTY}

Four main categories of approaches can be distinguished for dealing with spatial vagueness and imprecision. We first clarify the differences and relationships between terms related to vagueness and imprecision and how they appear in spatial data. For our classification of these terms we consider the previous attempts in [Parsons 1996, Worboys 1998].

We recognize two hierarchies of related concepts (see Figure 1). The first roots at the term imperfection, whereas the second roots at the concept of vagueness. Although semantically unrelated, these two concepts are related in practice due to the fact that imperfection can (and often does) lead to vagueness. It is important to note that these concepts are not exclusive, which means that objects can be classified into more than one concept at the same time.

The concept of imperfection can appear with two different faces: inaccuracy and imprecision. Inaccuracy is related to errors at the time of data capture. For example, the statement "the USA has a smaller area than Costa Rica" is inaccurate. Imprecision on the other hand deals with data that is not necessarily inaccurate but rather it is not determinate, often due to the finite representations available for spatial 


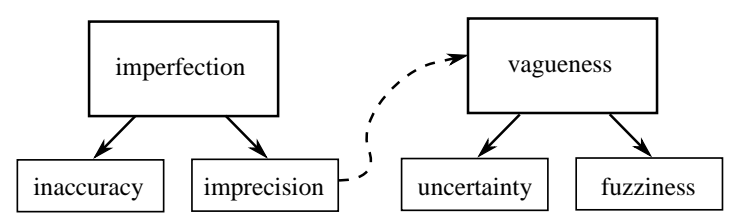

Figure 1: Hierarchy of concepts for imperfection and vagueness

objects. For example, the statement "Costa Rica is a small country" is imprecise as it describes a relative attribute and there is no inherent exact measurement of the small concept. Imprecision is sometimes considered as a type of vagueness not inherent to the objects that are described but rather derived from the data that is used to represent them.

Uncertainty describes a type of vagueness or indeterminacy that is associated to a crisp concept that is not known or cannot be measured precisely [Schneider 1999], for example the extension of underground iron ore deposits. On the other hand, fuzziness describes vagueness that is inherent to objects (i.e., their boundary, location, or extension is not precisely defined). Examples of vague objects include mountains, oceans, biotopes, and forests.

In the following, we proceed with different approaches that deal with spatial vagueness only in various ways.

\subsection{Extension of Exact Models into Three- Valued Logics}

The most popular approaches to handling spatial vagueness that utilize existing exact models for crisp spatial objects are the broad boundaries approach [Clementini and Felice 1996], the egg-yolk approach [Cohn and Gotts 1996], and the vague regions concept [Erwig and Schneider 1997]. These models extend the common assumption that boundaries of regions divide the plane into two sets (the set that belongs to the region, and the set that does not) with the notion of an intermediate set that is not known to certainly belong or not to the region. Thus we say that these models extend crisp models that operate on the Boolean logic (true, false) into models that handle uncertainty with a three-valued logic (true, false, maybe).

Two rather similar modeling approaches but with a rather different formal framework are the regions with broad boundaries approach [Clementini and Felice 1996] based on point set topology and the egg-yolk model [Cohn and Gotts 1996] based on a region connection calculus. Both approaches leverage crisp simple regions for representing spatial vagueness. An inner crisp simple region (the yolk) describes the zone of an uncertain region that definitely belongs to that region. The difference of a larger outer crisp simple region and the inner crisp simple region models the zone that maybe or maybe not belong to the uncertain region. 44 and 46 topological relationships respectively between two uncertain regions have been identified by the regions with broad boundaries approach and the egg-yolk model respectively.

The vague regions approach [Erwig and Schneider 1997] leverages complex regions possibly with several components and holes. A vague region is represented by two complex regions, one denoted as the kernel, the other as the boundary. The kernel represents the area that certainly belongs the region. The boundary represents the area that may or may not belong to the region (see Figure 2(b)). Vague regions are

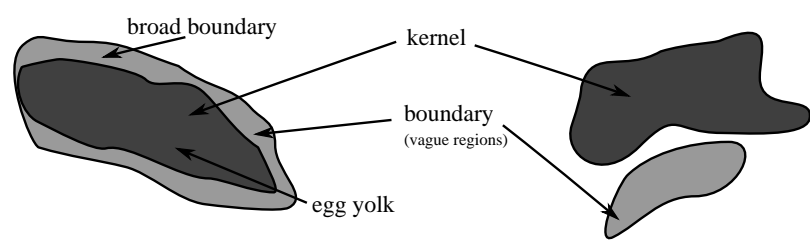

(a)

(b)

Figure 2: Examples of a broad boundary and eggyolk region $(a)$, and two vague regions $(a, b)$.

closed under well defined spatial set operations (i.e., geometric intersection, union, difference, complement). This is not the case in the previous two approaches. The vague regions approach is the precursor to VASA, presented in Section 3.

\subsection{Spatial Data Modeling with Rough Sets}

Rough set theory [Pawlak 1982] defines a rough set on the basis of a lower approximation and an upper approximation, which are both crisp sets. The lower approximation indicates the elements that certainly belong to the set whereas the upper approximation also includes those elements that may or may not belong to the set. At the core of rough set theory, indiscernibility relations between attributes of elements in the set are used to define the upper and lower approximations. These relations can be used to manipulate the granularity on which the approximations are established.

Worboys [Worboys 1998] uses rough sets for providing a basis for integrating and reasoning about multi-resolution spatial data, i.e., data that was captured at different resolutions but needs to be handled together. The authors in [Beaubouef and Petry 2002] use rough sets to model uncertainty in topological relationships between egg-yolk regions. In comparing the expressiveness of using the RCC model versus rough set theory, some relationships become indistinguishable using rough sets, while others can be specified with higher precision.

\subsection{Fuzzy Set Theoretic Approaches to Spatial Data Handling}

Fuzzy set based approaches attempt to model inherently vague spatial objects like a pollution cloud or a temperature zone. Much work has focused on the definition of data types designed for the treatment of fuzzy spatial objects. Fuzzy regions presented in [Altman 1994] assign membership to some property for every coordinate point within the (fuzzy) region. A formal definition of fuzzy points, fuzzy lines and fuzzy regions is included in [Schneider 1999]. A recent effort for the definition of a vague spatial algebra based on fuzzy sets is presented in [Dilo et al. 2004].

\subsection{Probabilistic Methods}

Probabilistic approaches for handling spatial vagueness such as that in [Finn 1993] model spatial objects on the basis of the probability of membership of an entity (i.e., point, area, object) in a set. This results in an expected membership which is based on the defined probability function.

\section{VAGUE SPATIAL ALGEBRA}

In this section, we describe our concept of a Vague Spatial Algebra (VASA). Its data types are specified in Section 3.1. Spatial set operations and metric operations are introduced 
in Section 3.2. Finally, the concept of vague topological predicates is briefly introduced in Section 3.3.

\subsection{Vague Spatial Data Types}

An important goal of VASA is to leverage existing definitions of crisp spatial concepts. We make use of a generic vague spatial type constructor $v$ that, when applied to any crisp spatial data type (i.e., point, line, region), renders a formal syntactic definition of its corresponding vague spatial data type. For any crisp spatial object $A$, we define its composition from three disjoint point sets, namely the interior $\left(A^{\circ}\right)$, the boundary $(\partial A)$ that surrounds the interior, and the exterior $\left(A^{-}\right)$[Schneider and Behr 2006]. We also assume a definition of the geometric set operations union $(\oplus)$, intersection $(\otimes)$, difference $(\ominus)$, and complement $(\boxminus)$ between crisp spatial objects.

Definition 1 Let $\alpha \in\{$ point, line, region $\}$. A vague spatial data type is given by a type constructor $v$ as a pair of equal crisp spatial data types $\alpha$, i.e.,

$$
v(\alpha)=\alpha \times \alpha
$$

such that for $w=\left(w_{k}, w_{c}\right) \in v(\alpha)$, holds:

$$
w_{k}^{\circ} \cap w_{c}^{\circ}=\varnothing
$$

We call $w \in v(\alpha)$ a (two-dimensional) vague spatial object with kernel part $w_{k}$ and conjecture part $w_{c}$. Further, we call $w_{o}:=\boxminus\left(w_{k} \oplus w_{c}\right)$ the outside part of $w$. For $\alpha=$ point, $v$ (point) is called a vague point object and denoted as vpoint. Correspondingly, for line and region we define $v$ (line) resulting in vline and $v$ (region) resulting in vregion.

Syntactically, a vague spatial object is represented by a pair of crisp spatial objects of the same type. Semantically, the first object denotes the kernel part that represents what certainly belongs to the object. The second object denotes the conjecture part that represents what is not certain to belong to the object. We require that the interiors of both underlying crisp objects are disjoint from each other. Figure 3 illustrates instances of a vague point, a vague line, and a vague region as objects of the data types defined above.

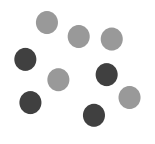

(a)

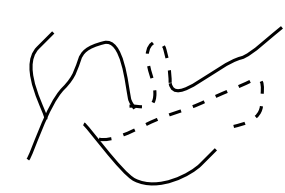

(b)

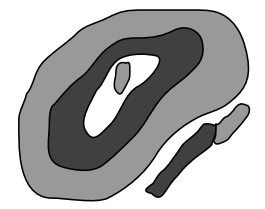

(c)
Figure 3: Examples of a vague point (a), a vague line (b) and a vague region (c). Kernel parts are symbolized by dark gray points, straight lines, and dark gray areas. Conjecture parts are symbolized by light gray point, dashed lines, and light gray areas.

\subsection{Vague Spatial Operations}

The vague spatial set operations that compute the union, intersection, and difference between two vague spatial objects leverage crisp spatial set operations. Syntactically, we define a function $h \in$ intersection, union, difference $\}$ as $h: v(\alpha) \times v(\alpha) \rightarrow v(\alpha)$. The complement operation is defined as complement $: v(\alpha) \rightarrow v(\alpha)$. Semantically, their generic (type independent) definition is achieved by considering the individual relationships between kernel parts, conjecture parts, and the outside part of the vague spatial objects involved in the operations. The result of each operation is computed using one of the tables in Table 1. For each operation the rows denote the parts of one object and the columns the parts of another, and we label them $k, c$, and $o$ to denote the kernel part, conjecture part, and outside part respectively. Each entry of the table denotes the intersection of kernel parts, conjecture parts, and outside parts of both objects, and the label in each entry specifies whether the corresponding intersection belongs to the kernel part, conjecture part, or outside part of the operation's result object.

\begin{tabular}{|c|c|c|c|}
\hline difference & $\begin{array}{lll}k & c & o\end{array}$ & & \\
\hline$k$ & $\begin{array}{lll} & c & k\end{array}$ & complement & $\begin{array}{lll}k & c & o\end{array}$ \\
\hline$c$ & $\begin{array}{lll}o & c & c\end{array}$ & & $\begin{array}{lll}o & c & k\end{array}$ \\
\hline
\end{tabular}

\begin{tabular}{r|rrrr|rrr} 
union & $k$ & $c$ & $o$ \\
\hline$k$ & $k$ & $k$ & $k$ & intersection & $k$ & $c$ & $o$ \\
\hline$c$ & $k$ & $c$ & $c$ & $k$ & $k$ & $c$ & $o$ \\
$o$ & $k$ & $c$ & $o$ & $c$ & $c$ & $c$ & $o$ \\
$o$ & $o$ & $o$ & $o$
\end{tabular}

Table 1: Components resulting from intersecting kernel parts, conjecture parts, and outside parts of two vague spatial objects with each other.

Each table from Table 1 can be used to generate an executable specification of the given crisp spatial operations. For each table, the specification will operate on the kernel parts and conjecture parts to result in a definition of its corresponding vague spatial operation. Following are such definitions as executable specifications of geometric set operations over crisp spatial objects:

Definition 2 Let $u, w \in v(\alpha)$, and let $u_{k}$ and $w_{k}$ denote their kernel parts and $u_{c}$ and $w_{c}$ their conjecture parts. We define:

$$
\begin{aligned}
\text { (i) } u \text { union } w:= & \left(u_{k} \oplus w_{k},\left(u_{c} \oplus w_{c}\right) \ominus\right. \\
& \left.\left(u_{k} \oplus w_{k}\right)\right) \\
\text { (ii) } u \text { intersection } w:= & \left(u_{k} \otimes w_{k},\left(u_{c} \otimes w_{c}\right) \oplus\right. \\
& \left.\left(u_{k} \otimes w_{c}\right) \oplus\left(u_{c} \otimes w_{k}\right)\right) \\
\text { (iii) } u \text { difference } w:= & \left(u_{k} \otimes\left(\boxminus\left(w_{k} \oplus w_{c}\right)\right),\left(u_{c} \otimes w_{c}\right) \oplus\right. \\
& \left.\left(u_{k} \otimes w_{c}\right) \oplus\left(u_{c} \otimes\left(\boxminus\left(w_{k} \oplus w_{c}\right)\right)\right)\right) \\
\text { (iv) complement } u:= & \left(\boxminus\left(u_{k} \oplus u_{c}\right), u_{c}\right)
\end{aligned}
$$

We have to prove formally the correspondence between these executable specifications and the tables in Table 1. These proofs can be found in [Pauly and Schneider 2004].

\subsection{Vague Topological Predicates}

For the definition of topological predicates between vague spatial objects (vague topological predicates), it is our goal to continue leveraging existing definitions of crisp spatial concepts, in this case topological predicates between crisp spatial objects. Topological predicates are used to describe purely qualitative relationships such as overlap and disjoint that describe the relative position between two objects and are preserved under continuous transformations.

For two vague spatial objects $A \in v(\alpha)$, and $B \in v(\beta)$ and the set $T_{\alpha \beta}$ of all crisp topological predicates between 
objects of types $\alpha$ and $\beta$ [Schneider and Behr 2006], the topological relationship between $A$ and $B$ is determined by the 4-tuple of crisp topological relationships $(p, q, r, s)$ such that $p, q, r, s \in T_{\alpha \beta}$ and:

$$
\begin{aligned}
& p\left(A_{k}, B_{k}\right) \wedge q\left(A_{k} \oplus A_{c}, B_{k}\right) \wedge \\
& r\left(A_{k}, B_{k} \oplus B_{c}\right) \wedge s\left(A_{k} \oplus A_{c}, B_{k} \oplus B_{c}\right)
\end{aligned}
$$

We define the set $V_{\alpha \beta}$ of all vague topological predicates between objects of types $v(\alpha)$ and $v(\beta)$. Due to inconsistencies that can exist between elements within each tuple, not all possible combinations result in 4-tuples that represent valid vague topological predicates in the set $V_{\alpha \beta}$. An example is the 4-tuple: (overlap $\left(A_{k}, B_{k}\right), \operatorname{disjoint}\left(A_{k}, B_{k} \oplus\right.$ $\left.B_{c}\right)$, disjoint $\left(A_{k} \oplus A_{c}, B_{k}\right)$, disjoint $\left.\left(A_{k} \oplus A_{c}, B_{k} \oplus B_{c}\right)\right)$. In this example overlap $\left(A_{k}, B_{k}\right) \Rightarrow A_{k}^{\circ} \cap B_{k}^{\circ} \neq \emptyset$ and $\operatorname{disjoint}\left(A_{k}, B_{k} \oplus B_{c}\right) \Rightarrow A_{k}^{\circ} \cap\left(B_{k} \oplus B_{c}\right)^{\circ}=\emptyset$. These two implications clearly contradict one another because according to the definition of the spatial union operation it holds that $B_{k}^{\circ} \subseteq\left(B_{k} \oplus B_{c}\right)^{\circ}$ and by the transitivity of set containment it is implied that $A_{k}^{\circ} \cap\left(B_{k} \oplus B_{c}\right)^{\circ} \neq \emptyset$. This directly contradicts disjoint $\left(A_{k}, B_{k} \oplus B_{c}\right)$.

In [Pauly and Schneider 2006], we present a method for identifying the complete set of vague topological predicates. At the heart of the method, each 4-tuple is modeled as a binary spatial constraint network (BSCN). The modeling is done by considering $A_{k}, B_{k}, A_{k} \oplus A_{c}$, and $B_{k} \oplus B_{c}$ as variables in the network, and $p, q, r$, and $s$ as constraints over the variables. The implicit containment relationships between $A_{k}$ and $A_{k} \oplus A_{c}$, and between $B_{k}$ and $B_{k} \oplus B_{c}$ are also modeled as constraints. Each BSCN is tested for pathconsistency which is used to check, via constraint propagation, that all original constraints are consistent.

For each type combination of vpoint, vline, and vregion, possibly thousands of predicates are recognized. As a result a further step of predicate clustering is needed to make these predicates accessible for querying.

Sets of 4-tuples are created into clustered vague topological predicates. Clusters can be defined by the user who specifies three rules for each cluster; one rule is used to determine whether the clustered predicate certainly holds between the objects, the second to determine if the cluster certainly does not hold, and the third to determine when the cluster maybe holds, but it is not possible to give a definite answer. This effectively symbolizes the three-valued logic that is central to our definition of vague spatial data types.

The specification of rules for each cluster can be performed at two different levels; the first is the crisp topological predicate level, where the criteria is specified on the basis of the values of $p, q, r, s$. For example, if $p, q, r, s$ represent crisp topological predicates between simple spatial objects, a cluster labeled Disjoint can be represented by the following three rules:

$$
\begin{aligned}
\operatorname{Disjoint}(A, B)=\text { true } & \Leftrightarrow \operatorname{disjoint}\left(A_{k} \oplus A_{c}, B_{k} \oplus B_{c}\right) \\
& \Leftrightarrow s=\operatorname{disjoint} \\
\operatorname{Disjoint}(A, B)=\text { false } & \Leftrightarrow \neg \operatorname{disjoint}\left(\left(A_{k}\right),\left(B_{k}\right)\right) \\
& \Leftrightarrow p \neq \operatorname{disjoint} \\
\operatorname{Disjoint}(A, B)=\text { maybe } & \Leftrightarrow \text { otherwise } \\
& \Leftrightarrow \neg(s=\text { disjoint } \vee p \neq \text { disjoint })
\end{aligned}
$$

Clustering rules can also be specified at the point set level. That is, the rules are defined on the basis of the emptiness (or non-emptiness) of the intersection between all combinations of interior $\left({ }^{\circ}\right)$, boundary $(\partial)$ and exterior $\left(^{-}\right)$of the crisp spatial objects that make up the vague spatial objects for which the cluster is defined. This type of specification allows for more general rules that are independent of the set of topological predicates that operate on the underlying crisp spatial objects. For example, the true rule for the cluster Disjoint can be specified more generally as:

$$
\begin{aligned}
\operatorname{Disjoint}(A, B)=\text { true } & \Leftrightarrow\left(\left(A_{k} \oplus A_{c}\right)^{\circ} \cap\left(B_{k} \oplus B_{c}\right)^{\circ}=\varnothing\right) \\
& \wedge\left(\left(A_{k} \oplus A_{c}\right)^{\circ} \cap \partial\left(B_{k} \oplus B_{c}\right)=\varnothing\right) \\
& \wedge\left(\partial\left(A_{k} \oplus A_{c}\right) \cap\left(B_{k} \oplus B_{c}\right)^{\circ}=\varnothing\right) \\
& \wedge\left(\partial\left(A_{k} \oplus A_{c}\right) \cap \partial\left(B_{k} \oplus B_{c}\right)=\varnothing\right)
\end{aligned}
$$

This rule implies that the vague spatial objects $A$ and $B$ are truly disjoint if none of their components have intersections of interiors or boundaries between each other.

\section{CONCEPTUAL ANALYSIS}

In this section, we analyze the advantages and disadvantages of existing concepts for handling spatial vagueness. We focus on models that follow the exact model approach, and explore how these compare specifically to rough set approaches. A direct comparison with fuzzy and probabilistic approaches does not make sense since the goals are rather different. Nevertheless, we provide some insight into the advantages and disadvantages of these models.

The conceptual design of VASA shows the clear goal of leveraging existing crisp concepts and is motivated by the following reasons. The first reason is to take advantage of existing robust concepts for handling crisp spatial objects. Second, at the conceptual level, the correctness of the definitions for vague concepts largely rests on the correctness of the defined crisp concepts; thus, we reduce the chance of errors in our definitions. An example is the definition of vague spatial operations in Definition 2 by an executable specification on the basis of crisp spatial operations. Third, the executable specification translates easily to the implementation level. Having an existing correct implementation of crisp spatial data types, their operations, and predicates, we can implement VASA by employing existing crisp spatial data types and executing operations on them. Figure 4 illustrates the architecture of implementing VASA on the basis of an existing crisp spatial algebra for databases. VASA needs no knowledge of the underlying DBMS that is used. Instead, it can simply make high level calls to the existing crisp algebra implementation.

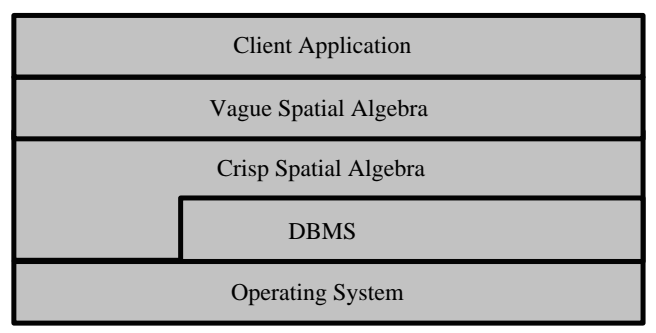

Figure 4: The architecture of a system implementing VASA.

Conceptually, VASA's goal of leveraging existing crisp concepts is not unique to the model itself but instead common to the models that follow the exact model approach. These models are generally considered appropriate for handling spatial imprecision and uncertainty. Similar to exact model based approaches, rough set based approaches are 
considered appropriate for handling imprecision and uncertainty. Thus, there is a natural tendency to compare both types of approaches. This is further implied by the application of rough set theory on top of an exact model based approach as done in [Beaubouef and Petry 2002].

When comparing the two types of approaches, it is important to consider the elements that make them seemingly different. The first is the concept of indiscernibility relations that exists in rough sets. This concept rests on an implicit function that defines the granularity with which the lower and upper approximation are defined. The separation of lower and upper approximations, or certain and uncertain parts of a spatial object, is not a feature of the object, as it is when using exact model based approaches, but instead a computed value that depends on a possibly subjective and domain specific function. This represents an advantage in cases where such a function is formally known and can even be adjusted if needed. It can be considered a disadvantage because of the lack of genericity due to the dependence on domain knowledge to define the function or if the function cannot be appropriately defined. The second element that differentiates both models is implementation. While exact model based approaches can use available implementations of their corresponding crisp spatial models so that their implementation requires less effort and is less errorprone, rough set based approaches require their own specific implementation.

At the heart of fuzzy set based approaches, a membership function is in charge of deciding to which degree each point or set of points belongs to a spatial object. The membership value does not describe the uncertainty of the point belonging to the object, but rather an actual degree of how much the point belongs to the object. Thus, fuzzy set based approaches are appropriate for dealing with fuzziness as an inherent property of the objects. In contrast, the function at the heart of probabilistic methods is used to describe the probability that a point or set of points belongs to an object. Thus, the probability function describes the degree of certainty that the point actually belongs to the object, which makes probabilistic approaches appropriate for modeling imprecision and uncertainty.

Fuzzy set theory has been recently considered the preferred option for handling spatial vagueness. Most approaches, like some of those mentioned in Section 2.3, focus on providing domain specific solutions. From an implementation standpoint, this provides better viability of an actual implementation but restricts the models to the domain they were designed for. The model in [Dilo et al. 2004] is implemented as an extension of GRASS, an open source GIS suite. This concept and implementation effectively simulates the extension of the three-valued logic concepts and implementation for VASA into an $n$-valued implementation.

A disadvantage with such an extension is the definition of spatial relationships, specifically topological relationships. It appears as though the extension to an $n$-valued logic would provoke a (possibly large) increase in the number of topological relationships between all type combinations of vague points, lines and regions.

\section{CONCLUSIONS}

In this paper, we have explored the most important approaches and models to modeling spatial vagueness and imprecision. We have focused on the definition of VASA as a point of comparison to establish the conceptual viability and potential for implementation of the different approaches.

The comparison and further analysis sheds light on advantages and disadvantages of each of the approaches. Many of the advantages are not necessarily contradicting across the different models and instead implicitly propose future work that would enable hybrid models that include the positive features of the different approaches. For example, it would be interesting to investigate the possible implementation of $n$-valued logic based approaches $(n>3)$ based on the VASA model. How would these models behave if $n$ is fixed, or if $n$ is variable?

\section{REFERENCES}

Altman, D. 1994. Fuzzy Set Theoretic Approaches for Handling Imprecision in Spatial Analysis. Int. Journal of Geographical Information Systems 8, 3, 271-289.

Beaubouef, T. And Petry, F. 2002. A Rough Set Foundation for Spatial Data Mining Involving Vague Regions . In IEEE Int. Conf. on Fuzzy Systems. IEEE Computer Society, $767-772$.

Clementini, E. And Felice, P. 1996. An Algebraic Model for Spatial Objects with Indeterminate Boundaries. In Geographic Objects with Indeterminate Boundaries. Taylor \& Francis, 153-169.

Cohn, A. G. And Gotts, N. M. 1996. The 'Egg-Yolk'

Representation of Regions with Indeterminate Boundaries. In Geographic Objects with Indeterminate Boundaries. Taylor \& Francis, 171-187.

Dilo, A., De By, R., And Stein, A. 2004. Definition and Implementation of Vague Objects. In Int. Symp. on Spatial Data Quality. 139-145.

Erwig, M. AND Schneider, M. 1997. Vague Regions. In 5thInt. Symp. on Advances in Spatial Databases. Springer-Verlag, 298-320.

Finn, J. T. 1993. Use of the Average Mutual Information Index in Evaluating Classification Error and Consistency. Int. Journal of Geographical Information Systems 7, 4, 349-366.

PARsons, S. 1996. Imperfect Information in Knowledge and Databases: A Survey of Current Approaches. IEEE Trans. on Knowledge and Data Engineering (TKDE) 8, 353-372.

PAuly, A. And Schneider, M. 2004. Vague Spatial Data Types, Set Operations, and Predicates. In East-European Conf. on Advances in Databases and Information Systems. 379-392.

Pauly, A. And Schneider, M. 2006. Topological Reasoning for Identifying a Complete Set of Topological Predicates between Vague Spatial Objects. In FLAIRS Conference. AAAI Press.

PAWLAK, Z. 1982. Rough sets. International Journal of Computer and Information Sciences, 341-356.

SCHNEIDER, M. 1999. Uncertainty Management for Spatial Data in Databases: Fuzzy Spatial Data Types. In Int. Symp. on Advances in Spatial Databases. Springer-Verlag, 330-351.

Schneider, M. AND BeHr, T. 2006. Topological Relationships between Complex Spatial Objects. ACM Trans. on Database Systems (TODS) 31, 39-81. Worboys, M. 1998. Imprecision in Finite Resolution Spatial Data. GeoInformatica 2, 3, 257-279. 